MS23-P04

\section{TEM-based analysis of the crystal structure of a Ge-rich layer sandwiched between spintronic $\mathrm{Fe}_{3} \mathrm{Si}$}

Holm Kirmse ${ }^{1}$, Bernd Jenichen², Elena Willinger ${ }^{3}$, Xing Huang ${ }^{3}$, Benedikt Haas ${ }^{1}$, Christoph T. Koch ${ }^{1}$

1. Humboldt University of Berlin, Dept. of Physics, Structure Physics and Electron Microscopy Group, Berlin, Germany

2. Paul Drude Institute, Berlin, Germany

3. Fritz Haber Institute, Berlin, Germany

email: holm.kirmse@physik.hu-berlin.de

Introduction

The magnetic properties of $\mathrm{Fe}_{3} \mathrm{Si}$ make it an excellent candidate for spintronic applications [1]. For a switchable device, two layers of $\mathrm{Fe}_{3} \mathrm{Si}$ have to be separated by a thin semiconducting interlayer. Ge was chosen for this interlayer having a nominal thickness of $3 \mathrm{~nm}$ only. Sharp interfaces were ensured by solid phase epitaxy [2] which is a two-step approach comprising of layer deposition at $150{ }^{\circ} \mathrm{C}$ and recrystallization at $300{ }^{\circ} \mathrm{C}$. From bulk crystals Fm $3 \mathrm{~m}$ space group is expected for $\mathrm{Fe}_{3} \mathrm{Si}$ and $\mathrm{Fd} 3 \mathrm{~m}$ for $\mathrm{Ge}$ [3].

Objectives

This work aims at both, crystal structure and composition analysis of the layer stack by transmission electron microscopy (TEM)-based techniques. In particular, nano-beam diffraction (NBD), high-angle annular dark-field atomic-resolution scanning TEM (HAADF ARSTEM) imaging and energy dispersive X-ray spectroscopy (EDXS) were applied for full crystallographic phase analysis.

Results

The generated crystallographic phase of $\mathrm{Fe}_{3} \mathrm{Si}$ perfectly corresponds to both, the expected crystal structure and the chemical composition. In contrast to that, superstructure reflections along [001] are observed for the nominal Ge layer. This hints to an ordering along [001]. Moreover, EDXS revealed a composition ratio of about 6:3:1 for Ge:Fe:Si. Both results point to an unexpected crystallographic phase of the interlayer. For gaining real space information, HAADF ARSTEM imaging was performed along [100] and [110] direction (see Fig. 1, center and right image, correspondingly). Bright spots correspond to atomic columns. The brightness depends on the mean atomic number of the individual column. Both projections consistently reveal ordering along [001]. Brighter spots are dedicated to Ge and weaker spots to Fe columns. Filled interlayers exhibit a larger distance along [001] of $\mathrm{z}=0.52$ compared to $\mathrm{z}=0.48$ for the empty layers.

Conclusion

The semiconductor interlayer is composed of $\mathrm{P} 4 \mathrm{~mm} \mathrm{Ge} \mathrm{Fe}_{2} \mathrm{Fe}$ (see Fig. 1 left). The deviation of the position of the atomic columns from the ideal positions at 0.50 and 0.75 is most likely due to the relaxation of strain introduced by the ordering.

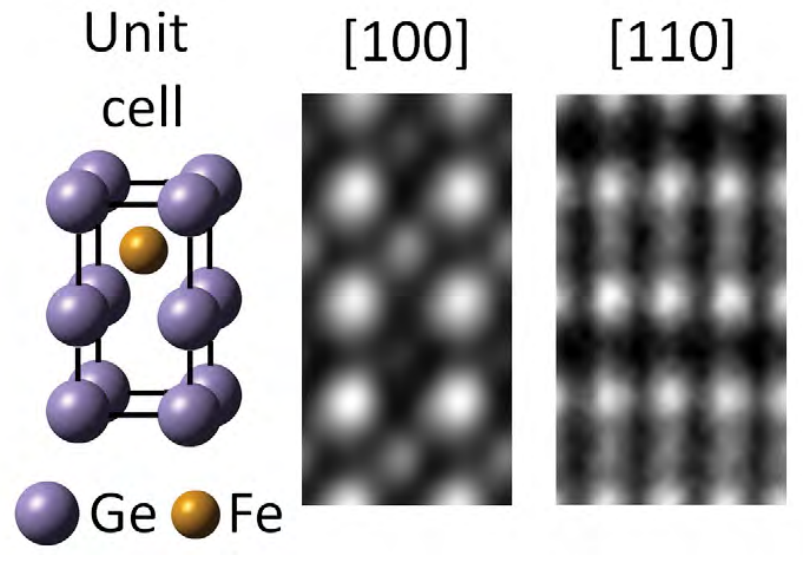

References:

[1] K. Hamaya et al., Appl. Phys. 113 (2013) 183713.

[2] S. Gaucher et al., Appl. Phys. Lett. 110 (2017) 102103.

[3] B. Jenichen et al., Phys. Stat. Sol. A 206 (2009) 1740.

Keywords: TEM, Spintronics, $\mathrm{Ge}_{2} \mathrm{Fe}$ on $\mathrm{Fe}_{3} \mathrm{Si}$ 